\title{
Spatial Distribution and Orientation of a Single Moving Dipole Computed in 12- lead ECGs of a Healthy Population Using a Spherically Bounded Model
}

\author{
Vito Starc ${ }^{1}$, Cees A Swenne ${ }^{2}$ \\ ${ }^{1}$ University of Ljubljana, Faculty of Medicine, Ljubljana, Slovenia \\ ${ }^{2}$ Cardiology Department, Leiden University Medical Center, Leiden, The Netherlands
}

\begin{abstract}
We investigated the possibility to assess moving dipoles (MD) from standard 12-lead ECGs in healthy humans to represent the electrical activity of the heart throughout the heart cycle. Since a moving dipole provides additional information when compared to a fixed dipole, we expect that different patterns of depolarization and/or repolarization in various types of cardiac diseases change the location of the dipole, thus providing information about the location of the pathological process.

We derived a model of the electric heart activity from the general solution of Laplace's equation, approximating the torso by a homogeneously conductive sphere, and representing the electrical forces by a dipole with variable location, orientation and strength. The model estimates (inverse solution) MDs from potentials at the 9 electrode locations of the standard 12-lead ECG, on a spherical surface adapted to a template torso.

Our study shows that application of a bounded spherical model using an MD for the description of the electrical activity of the heart consistently reproduces the measured surface potentials as provided by the 12-lead ECG.
\end{abstract}

\section{Introduction}

It is generally accepted that the electrical activity of the heart can be represented by a heart vector, representing the instantaneous dipole strength and direction [1,2]. This model has become the basis of contemporary vectorcardiography, which is relying on a heart vector fixed in space. Burger [3] found that the instantaneous amplitudes of voltages in the various ECG leads can be described by the dot product of the heart vector $\vec{H}$ and the lead vector $\vec{c}$, where direction and strength of a particular lead vector depend on the distances of the involved electrodes with respect to the heart, thus introducing a concept of a dynamic heart vector of which the location is fixed in space. In order to determine the 'heart vector', Gabor and Nelson [4] showed that distributed sources of electrical activity can be represented by equivalent dipoles and multipoles and derived a theorem that enabled their reconstruction. The concept of a moving dipole (MD) [5] was elaborated by Armoundas et al. [6,7] who showed that if spatially distributed bio-electrical sources are localized it is possible accurately to assess the dipole moment and its location of a 'single moving equivalent dipole (SEMD)' and if they are more spread the error might increase up to $20 \%$. They also introduced the optimization method for the assessment of SEMD.

Since a moving dipole provides additional information when compared to the a fixed dipole, we expect that if abnormal patterns of depolarization and/or repolarization occur in various types of cardiac diseases, the dipole will be in other locations than in a healthy heart, and that this location change provides important information about the location of the pathological process. Determination of the dynamic MD properties (location, strength and orientation) is therefore potentially important in the diagnosis of cardiac pathology.

Since the standard 12-lead ECG is the most prevalent form of electrocardiogram, we wondered whether it would be possible to assess the heart vector during the heart cycle as a single moving dipole (MD), computed from 12-lead ECGs in a healthy human population. We derived a model of the electric heart activity from the general solution of Laplace's equation, approximating the torso by a homogeneous conductive sphere, and representing the electrical forces by a dipole with variable location, orientation and strength. Surface potentials are proportional to the dot product of the dipole and the lead vectors of the 12-lead ECG. The model estimates (inverse solution) the MD from potentials at the 9 electrode locations of the standard 12-lead ECG, on the spherical surface adapted to a template torso. We used this model to find the spatial distribution and orientations of the MD within the thorax during the heart cycle and to determine its characteristics in a healthy population. Particular interest was paid to find the accuracy and reliability of the method. 


\section{Methods}

\subsection{A model for a dipole in a spherically bounded homogenous medium}

We derived an analytical expression for the potential $\varphi_{i k}$ at the point $\mathrm{P}_{\mathrm{i}}$ on a spherical surface, generated by a single dipole $\vec{D}_{k}$, located at the point $\mathrm{P}_{\mathrm{k}}$ within a homogeneous sphere with radius $R$ (Figure 1). The points $P_{k}$ and $\mathrm{P}_{\mathrm{i}}$ are given by radius vectors $\vec{r}_{k}$ and $\vec{r}_{i}$, respectively. The potential $\varphi_{i k}$ is expressed as the dot product of dipole vector $\vec{D}_{k}$ and lead vector $\vec{L}_{k i}$ which depends on the radius $\mathrm{R}$, the conductivity of the medium $\sigma$, on $\vec{r}_{k}$ and $\vec{r}_{i}$ :

with

$$
\varphi_{i k}=\vec{D}_{k} \cdot \vec{L}_{k i} \text {, }
$$

$\vec{L}_{k i}=\frac{1}{4 \pi \sigma R^{2}} \times\left(\frac{\vec{n}_{k i}}{\left|\vec{r}_{k i} / R\right|^{2}}+\frac{\vec{n}_{k i}-2\left(\vec{n}_{k} \cdot \vec{n}_{k i}\right) \vec{n}_{k}}{f^{3}\left|\vec{r}_{k i}^{*} / R\right|^{2}}+h\right)$

where

$h=-\frac{\vec{n}_{k}}{f}+\frac{\left(\mu+(f-\mu) / \sqrt{\left(1+f^{2}-2 \mu f\right)}\right)}{\sqrt{\left(1-\mu^{2}\right)}} \frac{\vec{n}_{k t}}{f}$

The first two terms in $\vec{L}_{k i}$ represent the contributions of two dipoles in an infinite homogenous medium: the object dipole $\vec{D}_{k}$ and its image dipole $\vec{D}_{k}$, the latter obtained by mirroring the object dipole over the spherical surface to point $\mathrm{P}_{\mathrm{k}}$, and the last term $h$ represents the part needed for making $\varphi_{i k}$ to be consistent with the general solution of Laplace`s equation for the surface potential of a dipole inside a homogenous conductive sphere. Due to space limitations the complete derivation cannot be described here. See Fig. 1 for explanation of variables and vectors.

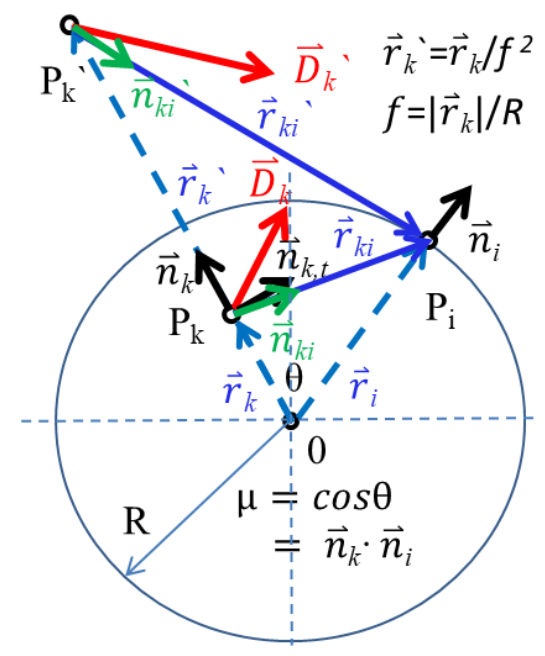

Figure 1. Representation of the moving dipole model in the plane through the points $\mathrm{P}_{\mathrm{k}}, \mathrm{P}_{\mathrm{i}}$, and the origin of the sphere. $\vec{D}_{k}, \vec{D}_{k}{ }^{*}$ : the object dipole and its image dipole (both in red); $\mathrm{P}_{\mathrm{k}}, \mathrm{P}_{\mathrm{k}}{ }^{`}$ and $\mathrm{P}_{\mathrm{i}}$ : locations of $\vec{D}_{k}, \vec{D}_{k}{ }^{`}$ and of the measuring electrode, given by radius vectors $\vec{r}_{k}, \vec{r}_{k}{ }^{N}$, and $\vec{r}_{i}$ (light blue dashed arrows); $\vec{r}_{k i}, \vec{r}_{k i}{ }^{\prime}$ : difference vectors $\vec{r}_{i}-\vec{r}_{k}$ and $\vec{r}_{i}-\vec{r}_{k}$, from the object and image dipoles to the location of the electrode (blue arrows); $\vec{n}_{k}, \vec{n}_{i}, \vec{n}_{k i}, \vec{n}_{k i}$ : unit vectors in the directions of $\vec{r}_{k}, \vec{r}_{i}$ (both black), and of $\vec{r}_{k i}$ and $\vec{r}_{k i}$ (both green); $\vec{n}_{k t}=\left(\vec{n}_{i}-\mu \vec{n}_{k}\right) / \sqrt{\left(1-\mu^{2}\right)}:$ the tangential unit vector at $\mathrm{P}_{\mathrm{k}}$ with $\mu=\vec{n}_{i} \times \vec{n}_{k}=\cos \theta ; \theta$ : angle between $\vec{n}_{k}$ and $\vec{n}_{i}$; $f=\left|\boldsymbol{r}_{k}\right| / \mathrm{R}$ : fractional distance (normalized for radius R) between the origin and the object dipole location.

\subsection{Approximation of the human thorax by a spherical homogenous conductor}

An adult torso was constructed using anatomical data of the adult human chest [8] that also provided locations of the ECG electrodes. Our model uses a sphere with radius $\mathrm{R}=15 \mathrm{~cm}$; this sphere was fitted to the anterior left surface of the chest with its center at the level of the chest electrode $\mathrm{C}_{3}$ in the transversal plane and $0.5 \mathrm{~cm}$ rightward and $1.5 \mathrm{~cm}$ backward from the center of the chest. The positions of the electrodes on the chest were mapped on the sphere. Our model uses electrode potentials V1..V6, VR, VL, and VF that are all determined with respect to Wilson's central terminal.

\subsection{Determination of the dipole strength and direction}

To find the dipole strength and direction, we minimize the error $F_{i k}=\varphi_{i k}-V_{i k}$ between the model potentials $\varphi_{i k}$ $=\vec{D}_{k} \cdot \vec{L}_{i k}$ and the measured potentials $V_{i k}$, in formula:

$$
\mathrm{F}_{i k}=\vec{D}_{k} \cdot \vec{L}_{i k}-V_{i k} \text {. }
$$

In the standard 12-lead ECG the 9 measured signals $\boldsymbol{V}_{i k}$ form an overdetermined system of 9 equations in which the 3 unknowns $D_{k x}, D_{k y}$, and $D_{k z}$ are linearly related to $V_{i k}$. To solve it, we applied principal component analysis to find $\vec{D}_{k}$ that minimizes

$$
\chi_{k}^{2}=\sum_{i=1}^{N i}\left|\vec{D}_{k} \cdot \vec{L}_{i k}-V_{i k}\right|^{2}
$$

The solution yields the directions and the strengths of dipole $\vec{D}_{k}$ for any given dipole location $\vec{r}_{i}$, with $\chi_{k}{ }^{2}$ the error of $\mathrm{V}_{i k}$ determination.

\subsection{Determination of the dipole location}

To find $\vec{D}_{k}$ and $\vec{r}_{k}$ that best describe the observed surface potentials, an inverse algorithm using an optimization method was applied. This was obtained by minimizing the objective function $\psi\left(\mathrm{r}_{\mathrm{k}}\right)$, defined as $\chi^{2}$ normalized with the sum of the squared potentials $V_{i k}$,

$$
\psi\left(\vec{r}_{k}\right)=\sum_{i=1}^{N i} \mathrm{~F}_{i k}^{2} / \sum_{i=1}^{N i} V_{i k}^{2}
$$

The square root of this function represents the root normalized mean square error (RNMSE, [6]). During optimization, $\vec{r}_{k}=\left(x_{k}, y_{k}, z_{k}\right)$ was varied and $\vec{D}_{k}$ 
determined at each iteration using (5), until reaching the optimal $\vec{r}_{k} . \vec{D}_{k}$ and $\vec{r}_{k}$ of the resulting MDs of each ECG sample $k$ were fed into (1) to get the reconstructed ECG signal, $\varphi_{i k}$. The reconstruction error was defined as the average RMS error between the reconstructed and the original ECG over all leads and over all QT intervals.

\subsection{Determination of MDs from ECG recordings}

Five-minute supine resting 12-lead high-frequent digital ECGs (Cardiax/Cardiosoft, sampling rate of 1 $\mathrm{kHz}, 300 \mathrm{~Hz}$ low pass filter) were recorded in 427 apparently healthy individuals (237 males, 190 females, age 20-69 years), excluding athletes. In 97 of these subjects, a second recording was obtained within one year, hence, the total number of ECGs considered in this study was 524. In each ECG, the separate beats and their positions were identified. The baseline was constructed by cubic interpolation through isoelectric points just before the QRS complex. For each ECG sample, 9 potentials (VR, VL, VF, V1..V6) were used to determine the corresponding MD (dipole strength, orientation and its location). For each of the first 20 beats a set of MDs were determined, from which a template MD set was obtained by determining median MDs over all beats, served for testing of quality and reproducibility of the method. Finally, the average RMS error between the reconstructed and the original ECG and the average RNMS error were computed as measure of quality of the method. Automatic analysis was performed using customized software (Delphi 5.0, Borland) on a PC [9].

To evaluate reproducibility, pairwise comparison was performed in subjects with two recordings $(\mathrm{N}=97)$, utilizing pairs of MDs belonging to each ECG to calculate the mutual distance $d r$ between MD locations and angular deviation $d \alpha$ due to difference in MD orientation. Finally, the mean distance $d r$ and the mean angle $d \alpha$ for segments and subjects were calculated. Small values of $d r$ and $d a$ would testify for good reproducibility of the method. Three kinds of reproducibility were studied. Beat-to-beat reproducibility was evaluated calculating distance $d r$ and angle $d \alpha$ between pairs MDs of the corresponding ECG beat and its template. Within-record reproducibility was considered as a measure of quality of ECG signal preparation (determination of the baseline and the QRS onset and offset) and was evaluated by comparing two parts of the same ECG recording (the same electrode positions), one obtained at the beginning of 5-min long recording and another 1-min before the end. Betweenrecordings reproducibility that could be connected with reproducibility of electrode positioning was evaluated by comparing two recordings from the same person.

The individual range of movement of MDs in 3-D space (MD ROM) was defined as the mean relative deviation of location of MDs during the QT interval from the average location in the QT interval. The latter was obtained by averaging MD locations over the QT interval.

To determine the spatial distribution of MDs in a healthy population, a 16-segment representation was used with 10 segments for the QRS complex, 3 for the ST segment and 3 for the $T$ wave. Specifically, the $1 \mathrm{~ms}$ MDs in the QRS complex of each individual beat were averaged over $5 \mathrm{~ms}$ segments, by displacing the next segment by an amount to fit the QRS duration by the first 10 segments. The $1 \mathrm{~ms}$ MDs in the ST segment and the T wave were averaged over $20 \mathrm{~ms}$. Those MDs of the ST segment started at 20, 40 and $60 \mathrm{~ms}$ after the $\mathrm{J}$ point, and those of the $\mathrm{T}$ wave were centered at its upslope, the peak and the downslope, respectively. The 16-segment properties of MDs were further averaged over all beats.

The spatial distribution of the MD locations and orientations for each ECG segment and each subject was quantified in voxels of $1.5 \mathrm{~cm} 3$. In each voxel, the mean MD orientation and its intra-voxel dispersion was determined.

\section{Results}

The mean \pm SD average RMS error was $37.1 \pm 18.2 \mu \mathrm{V}$ (5 and 95 percentiles 15-69). The relative error of the method, evaluated by the average RNMSE was $0.22 \pm 0.04$ (0.16-0.29).

Evaluation of the beat-to-beat, within-record and between-record reproducibility provided the average values of angular deviation $d a$ of $2.3 \pm 1.1^{\circ}, 8.5 \pm 3.8^{\circ}$ and $15.5 \pm 7.8^{\circ}$, respectively, and mutual distance $d r$ of $0.17 \pm 0.14,0.91 \pm 0.4$, and of $1.94 \pm 0.7 \mathrm{~cm}$, respectively.

The averaged MD ROM in the observed population was $2.77 \pm 0.43(2.1-2.75) \mathrm{cm}$ around the central point with 95-percentile range limited to $4.7 \mathrm{~cm}$. It was the highest at the end of the QRS complex (95-percentile range $5.6 \mathrm{~cm}$ ) and the first $\mathrm{ST}$ segment at the $\mathrm{J}$ point+20ms (95-percentile range $6.1 \mathrm{~cm}$ ).

The spatial distribution of MDs in a healthy population in a 16-segment representation showed that MDs were distributed over a relatively small volume, since there were only 34 voxels containing more than 4 samples. There was a small intra-voxel dispersion in the spatial MD orientation in each segment (mean dispersion of $16.5^{\circ}$ over all voxels).

\section{Discussion}

This study demonstrates that application of a bounded spherical model for the description of the electrical activity of the heart using a single moving dipole consistently reproduces measured surface potentials as provided by 12 lead ECG. The determination of MD location provided reproducible patterns in the movement 
of MDs with repeated measurements as evidenced by angular deviation $d \alpha$ and deviation distance $d r$.

Our finding that MDs obtained in a healthy population at similar time instants are spatially distributed over a relatively small volume and with a small intra-voxel orientation dispersion, allow us to use this ECG segment characteristic spatial distribution of the MD locations and orientations as the referential one that for identification of cardiac diseases with different distributions, as we expect in case of acute myocardial ischemia and in many different conditions.

Our beat-to-beat reproducibility of $d r$ of $0.17 \pm 0.14 \mathrm{~cm}$ is comparable to that in the study of Armoundas et al [7] who reached an accuracy between 1 and $3 \mathrm{~mm}$, and is small enough compared to the average MD ROM of 2.77 $\mathrm{cm}$ for a reliable detection of changes in MD movement.

The average RMS error was around $37 \mu \mathrm{V}$, representing $15 \%$ of the mean RMS voltage of $260.2 \pm 78.9 \mu \mathrm{V}$. The corresponding average RNMSE 0.22 was therefore considerably bigger than that of the relative RMS error of 0.15 . The difference arises in the process of averaging: whereas in the first (0.15), both average RMS values were determined initially and divided, in the second (0.22) relative error was first calculated per each sample and then averaged. The individual variation of both errors was considerable and highest in those parts of the ECG signal with low amplitudes $(<50 \mu \mathrm{V})$ and sometimes also at the end of the QRS complex and during T wave.

This finding demonstrates that the method becomes less reliable in ECGs with a low amplitude ST segment. Though the ST segment is an important clinical target for diagnosis of acute myocardial ischemia, ST segment amplitudes are usually larger than $100 \mu \mathrm{V}$, providing thus a much lower RNMSE.

In evaluating the reproducibility of MD determination, three different comparisons were performed to identify the processes responsible for increased values of $d \alpha$ and $d r$. When comparing beat-to-beat to within-record and between-recording reproducibilities we found an increasing trend in $d \alpha$ from around $2.5^{\circ}$ to $8^{\circ}$ and $15^{\circ}$ and in $d r$ from around $0.2 \mathrm{~cm}$ to 1 and $2 \mathrm{~cm}$, which reveals a large increase of both $d \alpha$ and $d r$ in the first step. Since within-a-record reproducibility incorporates beat-to-beat reproducibility and is connected with the pre-processing of the ECG signal, it suggests that the determination of baseline, the QRS onset and offset might be responsible for lowering of reproducibility. Though the accuracy of the delineation of the QRS complex does not interfere with the determination of MDs, any shifting of the QRS onset in one of the recording with respect to another may lead to comparison of mismatched MD and producing false positive result. In case of rapid changes in MDs properties during the QRS complex, mismatching of MD pairs could be responsible for decreased reproducibility even when delineation accuracy is below $10 \mathrm{~ms}$ as was the case in our analysis. Lastly, between - recordings reproducibility that incorporates the first two reproducibilities is sensitive to the position of surface electrodes and may reflect physiological or other changes of a subject that had occurred between two measurements. As the exactness of position of body surface electrodes influences the quality of determination of MDs, it could be used in detection of misplaced electrodes [9].

There are some other sources of error in our method. Our model ignores boundary conditions, inhomogeneities in tissue conductivity and realistic anatomic geometry. The model requires the exact position of body surface electrodes in 3-D space, and its accuracy decreases when concerning spread dipolar sources, such as may occur during the $T$ wave. As our model is insensitive to the presence of multipoles that might be connected with the spread dipolar sources, their presence might have also increased the RMS error.

\section{References}

[1] Einthoven W. The different forms of the human electrocardiogram and their signification. Lancet 1912: 853-61.

[2] Man S, Maan AC, Schalij MJ, Swenne CA. Vectorcardiographic diagnostic \& prognostic information derived from the 12-lead electrocardiogram: Historical review and clinical perspective. J Electrocardiol 2015; 48: 463-475.

[3] BurgerHC, Milaan JB. Heart vectors and leads; geometrical presentation. Br H J 1948; 10: 229-33.

[4] Gabor D, Nelson CV. Determination of the resultant dipole of the heart from measurements on the body surface. J Appl Phys 1954; 25: 413-416.

[5] Arthur RM, Geselowitz DB, Briller SA, Trost RF: The path of the electrical center of the human heart determined from surface ECGs. J Electrocardiol 1971; 4:(1) 29-33.

[6] Armoundas AA, Feldman AB, Sherman DA, Cohen RJ. Applicability of the single equivalent point dipole model to represent a spatially distributed bio-electrical source. Med Biol Eng Comput 2001; 39:562.

[7] Armoundas AA, Feldman AB, Mukkamala R, Cohen RJ.A single equivalent moving dipole model: An efficient approach for localization of sites of origin of ventricular electrical activation. Ann Biomed Eng 2003; 31: 564.

[8] Dawoud F, Wagner GS, Moody G, Horacek BM. Using inverse electrocardiography to image myocardial infarction-reflecting on the 2007 PhysioNet/Computers in Cardiology Challenge. J Electocardiol 41(6):630-5.

[9] Starc V. Could determination of equivalent dipoles from 12lead ECG help in detection of misplaced electrodes. Computing in Cardiology 2011; 38:445-448.

Address for correspondence.

Vito Starc, MD, $\mathrm{PhD}$

Ljubljana University, Faculty of Medicine

Zaloska 4

SI 1104 Ljubljana, Slovenia

E-mail: vito.starc@mf.uni-lj.si. 